\title{
Prevalence of Chondral Lesions in Knee Arthroscopy
}

\author{
Pál Fodor ${ }^{1,2}$, Árpád Sólyom ${ }^{3}$, Adrian Ivănescu 4 , Raluca Fodor ${ }^{5}$, Tiberiu Bățagă³ \\ ${ }^{1}$ Clinic of Orthopedics and Traumatology, Clinical County Emergency Hospital Mureș, Tîrgu Mureş, Romania \\ 2 "Ovidius" Clinical Hospital, Constanța, Romania \\ 3 Department of Anesthesiology and Intensive Care, University of Medicine and Pharmacy, Tîrgu Mureş, Romania \\ 4 Department of Orthopedics and Traumatology, University of Medicine and Pharmacy, Tîrgu Mureş, Romania \\ 5 Department of Anatomy, University of Medicine and Pharmacy, Tîrgu Mureş, Romania
}

\section{CORRESPONDENCE \\ Árpád Sólyom \\ Str. Gheorghe Marinescu 38 \\ 540139 Tîrgu Mureș, Romania \\ Tel: +40740695460 \\ E-mail: solyomarpad@yahoo.com}

\section{ARTICLE HISTORY}

Received: February 2, 2018

Accepted: March 22, 2018
Pál Fodor • Str. Gheorghe Marinescu nr. 50, 540136 Tîrgu Mureș, Romania. Tel: +40 265212111 Adrian Ivănescu • Str. Gheorghe Marinescu nr. 38 540139 Tîrgu Mureș, Romania. Tel: +40 265215551 Raluca Fodor • Str. Gheorghe Marinescu nr. 50 , 540136 Tîrgu Mureș, Romania. Tel: +40 265212111

Tiberiu Bătagă • Str. Gheorghe Marinescu nr. 38 540139 Tîrgu Mures, Romania. Tel: +40 265215551

\begin{abstract}
Background: Chondral and osteochondral injuries of the knee joint are undervalued in subjects undergoing orthopedic surgery. Chondral lesions are difficult to diagnose as they do not present specific clinical signs. The objective of the study was to establish the prevalence of cartilage injuries in patients undergoing arthroscopy of the knee for knee pain or instability. Materials and methods: A retrospective study was conducted on 355 consecutive knee arthroscopies. Chondral lesions were found in 247 (69.6\%) cases. Regarding their location, chondral lesions were more likely to be located on the medial femoral condyle (53.8\%), while lesions on the lateral femoral condyle alone $(0.8 \%)$ were the least frequent ones. Chondral injuries were frequently found with associated articular findings such as meniscal lesions (56.68\%) and anterior cruciate ligament tears (2.84\%) or both menisci and anterior cruciate ligament tear (15.38\%). The ICRS grade II cartilage lesions were most frequently diagnosed (56.3\%) while grade IV was the least frequent type (6.9\%). Conclusions: Chondral damage is frequently diagnosed during knee arthroscopy, present in more than $50 \%$ of the arthroscopies performed for various indications, and an important part of cartilage injuries were linked with other intraarticular findings, such as anterior cruciate ligament tear and medial meniscus tear.
\end{abstract}

Keywords: chondral injury, knee arthroscopy, ICRS classification

\section{INTRODUCTION}

Chondral and osteochondral damage is frequently encountered in current orthopedic clinical practice. The articular chondral tissue is an extremely specialized type of connective tissue of the diarthrodial joints that facilitates the transmission of loads with a low frictional coefficient, but with a limited capacity to regenerate, even following minor injuries. ${ }^{1-3}$ It also provides a smooth, lubricated surface for the articulation with a low-friction gliding surface, which none of the artificial constructs have been able to substitute with success. ${ }^{4}$

The clinical signs of chondral injuries have low specificity and predictive capacity, thus making their diagnosis difficult to achieve. Subjects who present 
chondral damage might present symptoms that are more specific to meniscal lesions. However, if left untreated, cartilage lesions of the knee can cause premature arthritis and can lead to impaired joint function and decreased quality of life. ${ }^{3,4}$

Being an accurate diagnostic and treatment method for disorders of the knee joint, the minimally invasive arthroscopy has become the gold standard procedure for investigation of the knee, with a diagnostic specificity of over $90 \% .3,5$

Damage of the chondral tissue in the joints is an underestimated issue in subjects who undergo orthopedic surgery for various conditions. Current data states that there is a proved incidence of 60 to 66 percent of cartilage injuries discovered during knee arthroscopies, regardless of the surgical indication. ${ }^{6-9}$

The aim of the study was to establish the prevalence, the type, and the localization of cartilage injuries in patients undergoing arthroscopy of the knee and to establish what their relationship is to other injuries.

\section{MATERIALS AND METHODS}

We conducted a retrospective observational study on 444 consecutive knee arthroscopies performed between 2014 and 2016, in the "Ovidiu" Clinical Hospital, Constanța, Romania.

The approval of the institutional review committee of the private hospital where the procedures had been conducted was obtained prior to the conduction of the study. Patient data was obtained from the medical files of the enrolled study subjects.

The inclusion criteria consisted in patients who underwent arthroscopy of the knee for painful knee joint, and with impaired articular function, loose body sensation, meniscal tears, or anterior cruciate ligament reconstruction.

The exclusion criteria consisted in the presence of prior major knee injury, inflammatory arthritis or infectious arthritis in the medical history of the patients, as well as subjects aged under 18 years or over 60 years.
TABLE 1. Patient distribution according to age

\begin{tabular}{lcc}
\hline Age group (years) & Incidence $(\mathbf{n})$ & $\%$ \\
\hline Under 30 & 70 & 19.7 \\
$31-40$ & 84 & 23.7 \\
$41-50$ & 105 & 29.6 \\
$51-60$ & 96 & 27.0 \\
TOTAL & 355 & 100.0 \\
\hline
\end{tabular}

The arthroscopies were conducted by two surgeons specialized in orthopedics and trauma, with an experience in practicing arthroscopic surgery for a minimum of 5 years. We extracted data concerning chondral damage (grade, site, status of adjacent chondral tissue), associated joint damage, as well as information on the surgical procedure, as they were noted after each arthroscopy in patient records by the operating surgeon. The grade of the lesion was assessed according to the International Cartilage Repair Society (ICRS) classification system. ${ }^{10}$

The data were collected from patients' medical files and were processed using Microsoft Excel and SPSS Statistics version 17.0 (IBM, New York, USA). Statistical analysis included frequencies, cross-tabs, and associations of the studied variables. Those associations were tested using likelihood ratio and a significance level of 0.05 .

\section{RESULTS}

A total of 444 consecutive knee arthroscopies performed between 2014 and 2016 were analyzed, and 355 patients were enrolled in the study as they did not present any of the major exclusion criteria. The mean age of the study population was $42.19 \pm 11.68$ years, ranging between 18 60 years, as presented in Table 1 .

Chondral lesions were found in $247(69.6 \%)$ cases, and their frequency increased with age $(\mathrm{p}<0.001)$ (Table 2$)$.

Regarding their location, chondral injuries were more frequent at the level of the medial femoral condyle (53.8\%), while lesions on the lateral femoral condyle alone $(0.8 \%)$ were the least frequent ones (Table 3).

TABLE 2. The distribution of chondral lesions according to age

\begin{tabular}{lcccc}
\hline & $\begin{array}{c}\text { Under } \mathbf{3 0} \\
\mathbf{n ~ ( \% )}\end{array}$ & $\begin{array}{c}\mathbf{3 1 - 4 0} \\
\mathbf{n ~ ( \% )}\end{array}$ & $\begin{array}{c}\mathbf{4 1 - 5 0} \\
\mathbf{n ~ ( \% )}\end{array}$ & $\begin{array}{c}\mathbf{5 1 - 6 0} \\
\mathbf{n}(\%)\end{array}$ \\
\hline Without chondral lesions & $57(81.42 \%)$ & $42(50 \%)$ & $9(8.57 \%)$ & $0(0 \%)$ \\
With chondral lesions & $13(18.57 \%)$ & $42(50 \%)$ & $96(91.42 \%)$ & $96(100 \%)$ \\
Total & 70 & 84 & 105 & 96 \\
\hline
\end{tabular}


TABLE 3. Anatomical distribution of chondral lesions

\begin{tabular}{lcc}
\hline Location & Incidence (n) & $\%$ \\
\hline Medial femoral condyle & 133 & 53.8 \\
Lateral femoral condyle & 2 & 0.8 \\
Patellae & 24 & 9.7 \\
Medial + lateral femoral condyle & 24 & 9.7 \\
Medial femoral condyle + trochlea & 3 & 1.2 \\
Medial femoral condyle + patellae & 38 & 15.4 \\
Medial + lateral femoral condyle + patellae & 4 & 1.6 \\
Medial + lateral femoral condyle + patellae + trochlea & 2 & 0.8 \\
Lateral femoral condyle + patellae & 2 & 0.8 \\
Patellae + trochlea & 3 & 1.2 \\
Medial femoral condyle + trochlea + patellae & 12 & 4.9 \\
TOTAL & 247 & 100.0 \\
\hline
\end{tabular}

TABLE 4. Distribution of other concomitant arthroscopic findings

\begin{tabular}{lcc}
\hline & Incidence (n) & $\%$ \\
\hline Chondral lesions alone & 62 & 25.10 \\
Chondral lesions + meniscal injury & 140 & 56.68 \\
Chondral lesions + anterior cruciate ligament tear & 7 & 2.84 \\
Chondral lesions + both & 38 & 15.38 \\
TOTAL & 247 & 100.0 \\
\hline
\end{tabular}

Chondral injuries were frequently associated with other joint injuries, such as meniscus lesions $(56.68 \%)$ and anterior cruciate ligament tear $(2.84 \%)$, or both menisci and anterior cruciate ligament tear (15.38\%). The distribution of other concomitant arthroscopic findings is shown in Table 4.

Grade II according to the ICRS classification system was the most frequent grade of cartilage injury (56.3\%), while grade IV was the least frequent one $(6.9 \%)$ ( $\mathrm{p}$ $<0.001$ ) (Table 5).

\section{DISCUSSIONS}

The present study confirms that joint cartilage lesions are commonly encountered in a young adult population. This study showed that from patients undergoing knee arthroscopy for various indications, $69.6 \%$ suffered at least one type of cartilage pathology.

Similar results have previously been published by other authors in larger study populations. Curl et al. reported a rate of chondral damage of $63 \%$ in a study that included 31,516 subjects. $^{7}$ Hjelle et al. found a $61 \%$ incidence of cartilage or osteochondral injuries when analyzing 1,000 consecutive patients undergoing arthroscopies, ${ }^{8}$ while
Aroen et al. found that $66 \%$ of 993 patients undergoing knee arthroscopies presented associated cartilage disorders. ${ }^{11}$ In another study that included a large number of patients (5,114 knee arthroscopies), Widuchowski et al. found chondral lesions in $57.3 \%$ of cases, ${ }^{12}$ but Figueroa et al. reported a lower rate of chondral tissue defects, in $41.16 \%$ of cases (82 out of 190 subjects who underwent knee arthroscopy). ${ }^{13}$

The rate of cartilage lesions fluctuated from 60 to $70 \%$ in most of the studies, their prevalence being concordant to our results.

In the present study, the most frequent site for cartilage lesions was found to be at the level of the medial femoral

TABLE 5. Type of chondral lesions according to the ICRS classification

\begin{tabular}{lcc}
\hline ICRS Type & Incidence & $\%$ \\
\hline I & 64 & 25.9 \\
II & 139 & 56.3 \\
III & 27 & 10.9 \\
IV & 17 & 6.9 \\
TOTAL & 247 & 100 \\
\hline
\end{tabular}


condyle (53.8\%), followed by the patella (9.7\%). The distribution of chondral lesions according to their anatomical location found in our study was similar to other studies on the subject.

Hjelle et al. reported that $58 \%$ of cartilage defects were located at the level of the medial femoral condyle, $11 \%$ in the patella, $11 \%$ in the lateral region of the tibial plateau, $9 \%$ in the lateral femoral condyle, and $5 \%$ in the medial part of the tibia. ${ }^{14}$ Widuchowski et al. found that the patellar area (36\%) and the medial femoral condyle (34\%) were the most common locations of chondral tissue injuries, while the medial region of the tibial surface (6\%) presented the lowest frequency. ${ }^{12}$ Correspondingly, Aroen et al. found that $43 \%$ of cartilage injuries were located in the medial femoral condyle, and $23 \%$ in the patellar region. ${ }^{11}$ Similarly, Curl et al. found the most common locations of damage in the articular cartilage at the level of the medial femoral condyle (32\%) and patella (21\%). ${ }^{7}$

In our study, the distribution of cartilage injuries within different parts of the knee joint was also in accordance with the studies referred above, but we found a higher percentage of non-isolated cartilage lesions (60.68\%).

We found full-thickness lesions (ICRS grade III-IV) in only a small percentage (17.2\%) of the analyzed knees. In contrast, Bikash et al. found that grade III, according to the Outerbridge classification, was the most common grade of chondral lesions ( $46 \%$ of cases), followed by grade IV ( $22 \%$ of cases). ${ }^{3}$ Our results were also in disagreement to the results of Hjelle et al. (14\% grade I, $26 \%$ grade II, $55 \%$ grade III, $5 \%$ grade IV lesions) and Curl et al. (9.7\% grade I, $28.1 \%$ grade II, $41.0 \%$ grade III, $19.2 \%$ grade IV lesions). 7,14

Cartilage defects are commonly associated with other pathological conditions of the joint such as anterior cruciate ligament ruptures and meniscal injuries. In our study, associated injuries of the medial meniscus were diagnosed in the highest proportion of patients $(56.68 \%)$, followed by both meniscus and anterior cruciate ligament tears (15.38\%). Similarly to our results, current studies show that frequently associated lesions include the medial meniscus and anterior cruciate ligament tear. ${ }^{10}$

\section{Study limitations}

One of the major limitations of the current study is its retrospective nature. Other study limitations include the rela- tively small number of included subjects (355 patients) and the existence of bias in collecting the necessary data from the patients' files, which may have adversely impact the reliability of our results.

\section{CONCLUSIONS}

Chondral injuries are frequently encountered in knee arthroscopy and are present in more than half of the arthroscopies performed. An important part of cartilage injuries is accompanying other intra-articular lesions, including the anterior cruciate ligament tear and medial meniscus tear. Based on these results, it is recommended to have the necessary resources at disposal for at least one cartilage repair technique during a knee arthroscopy, due to an increased likelihood of encountering previously undiagnosed chondral damage.

\section{CONFLICT OF INTEREST}

Nothing to declare.

\section{REFERENCES}

1. Buckwalter JA, Mankin HJ. Articular cartilage: Tissue design and chondrocyte-matrix interactions. Instr Course Lect. 1998;47:477-486.

2. Caplan Al, Elyaderani M, Mochizuki Y, et al. Principles of cartilage repair and regeneration. Clin Orthop. 1997;342:254-269.

3. Bikash KC, Lamichhane AP, Mahara DP. Prevalence of Chondral Lesion in Knee Arthroscopy. Austin J Trauma Treat. 2016;3:1012.

4. Bhosale AM, Richardson JB. Articular cartilage: structure, injuries and review of management. British Medical Bulletin. 2008;87:77-95.

5. Tait GR, Maginn P, Macey AC, et al. Unnecessary arthroscopies. Injury 1992;23:555-556

6. Aroen A, Loken S, Heir S, et al. Articular cartilage lesions in 993 consecutive knee arthroscopies. Am J Sports Med. 2004;32:211-215.

7. Curl WW, Krome J, Gordon ES, et al. Cartilage injuries: a review of 31,516 knee arthroscopies. Arthroscopy. 1997;13:456-460.

8. Hjelle K, Solheim E, Strand T, et al. Articular cartilage defects in 1,000 knee arthroscopies. Arthroscopy. 2002;18:730-734.

9. Shah MR, Kaplan KM, Meislin RJ, et al. Articular cartilage restoration of the knee. Bull NYU Hosp Jt Dis. 2007;65:51-60.

10. Brittberg M, Winalski C. Evaluation of cartilage injuries and repair. J Bone Joint Surg Am. 2003;85:58-69.

11. Aroen A, Deryk G, Jones DG, et al. Arthroscopic diagnosis and treatment of cartilage injuries. Sports Med Arthrosc Rev. 1998;6:31-40.

12. Widuchowski W, Kusz D, Widuchowski J, et al. Analysis of articular cartilage lesions in 5114 knee arthroscopies. Chir Narzadow Ruchu Ortop Pol. 2006;71:117-121.

13. Figueroa D, Calvo R, Vaisman A. Knee Chondral Lesions: Incidence and Correlation between Arthroscopic and Magnetic Resonance Findings. Arthroscopy: The Journal of Arthroscopic and Related Surgery. 2007;23:312-315.

14. Hjelle K, Solheim E, Strand T, et al. Articular cartilage defects in 1,000 knee arthroscopies. Arthroscopy. 2002;18:730-734. 